\title{
How surgeon and pathologist cooperation may drive toward a more efficient nodes harvesting in gastric cancer surgery
}

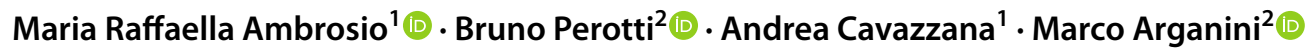

Received: 2 March 2021 / Accepted: 11 March 2021 / Published online: 7 May 2021

(C) Italian Society of Surgery (SIC) 2021

The down-staging of gastric cancer (GC) has recently gained particular attention. The phenomenon is mainly due to an inappropriate harvesting of lymph nodes (LNs). Standard treatment for GC includes curative gastrectomy plus D2 lymphadenectomy with a minimum number of $16 \mathrm{LNs}$ required for an appropriate $\mathrm{N}$ staging [1, 2]. However, the surgeon's technical skills, the pathologist's experience, and other unavoidable conditions may result in less than $16 \mathrm{LNs}$ examined. This has been deemed inadequate as it brings to pathological under staging, stage migration and poorer longterm clinical outcome due to incorrect treatment planning [3]. Moreover, it has been demonstrated that the number of dissected LNs represents and independent prognostic factor [4]. Removal of higher number ( $>30$ ) of LNs is helpful to enhance the rate of curative resection and reduce the incidence of local recurrence, thus improving the overall survival rate [5].

In Japan and in many specialized Western Institutions, LNs sampling is generally performed by the surgeon itself on the fresh specimen. In some non-specialized Western centers, it is generally achieved from formalin-fixed (FF) samples by pathologist during the grossing procedure; although this method is time-consuming, small LNs $(1.0 \mathrm{~mm})$ may still be missed [5]. Therefore, the hot question of how to accomplish a complete LNs harvesting still remains, as most of the procedures proposed and applied so far were often disappointing $[6,7]$. This results in the increasing needing of a standardized and easily applicable operative protocol.

The aim of this pivotal study was to standardize a procedure for on site macroscopic evaluation and sampling of

Marco Arganini

marco.arganini@uslnordovest.toscana.it

1 Pathology Unit, Azienda Ospedaliera Toscana Nord Ovest, Pisa, Italy

2 Surgery Unit, Ospedale Unico Versilia, Azienda Ospedaliera Toscana Nord Ovest, Via Aurelia n. 335, 55049 Lido di Camaiore, Italy
LNs according to the Japanese Gastric Cancer Association (JGCA) [8]. The procedure was carried out by a surgeon/ pathologist team in the operating room (OR). This with the ultimate goal to harvest the highest number of LNs detectable, thus providing a correct ratio between metastatic and total LNs collected and finally providing a precise staging and a tailored therapeutic approach.

The prospective phase of this study has started soon after the early wave of SARS-COV2 pandemic. We enrolled the first three patients presenting to the Surgery Unit of Ospedale Unico della Versilia with a diagnosis of GC according to the last WHO. The patients were 2 males and 1 female with a mean age of 76.3 years. All patients underwent a complete staging and a sub-total gastric resection followed by D1 or D2 nodes dissection depending on clinical conditions and tumor staging. All procedures were carried out under similar standardized principles by the same surgeon with years of experience in gastro-intestinal surgery. A pathologist, trained in gastric malignancies, was in the OR for on site immediate macroscopic evaluation and dissection of fresh fat tissue to retrieve LNs, according to the JGCA protocol. Adipose tissue was detached en bloc from the stomach wall, left gastric artery, hepatic peduncle, common hepatic artery, proximal splenic artery and splenic hilus, and coeliac axis (Fig. 1a, b). Next, the fat tissue was scraped off with scissors, separating blood vessels, nerves, and lymph nodes. The surgeon carefully indicated to the pathologist the blood vessels course and branching. Pathologist performed meticulous dissection of the fat tissue to retrieve LNs focusing mainly along the vessel tiers. After the visual phase, the remaining LNs were checked by tissue palpation. Each $\mathrm{LN}$ was picked up and mapped one by one. Later, the surgical specimens (including stomach, harvested lymph nodes and the remaining adipose tissue of the different nodal stations) were FF in separate boxes and sent out to the pathology lab for the final examination. After the fixation, the adipose tissue of the nodal stations was again reviewed by means of visualization and palpation to identify eventually missed LNs. 


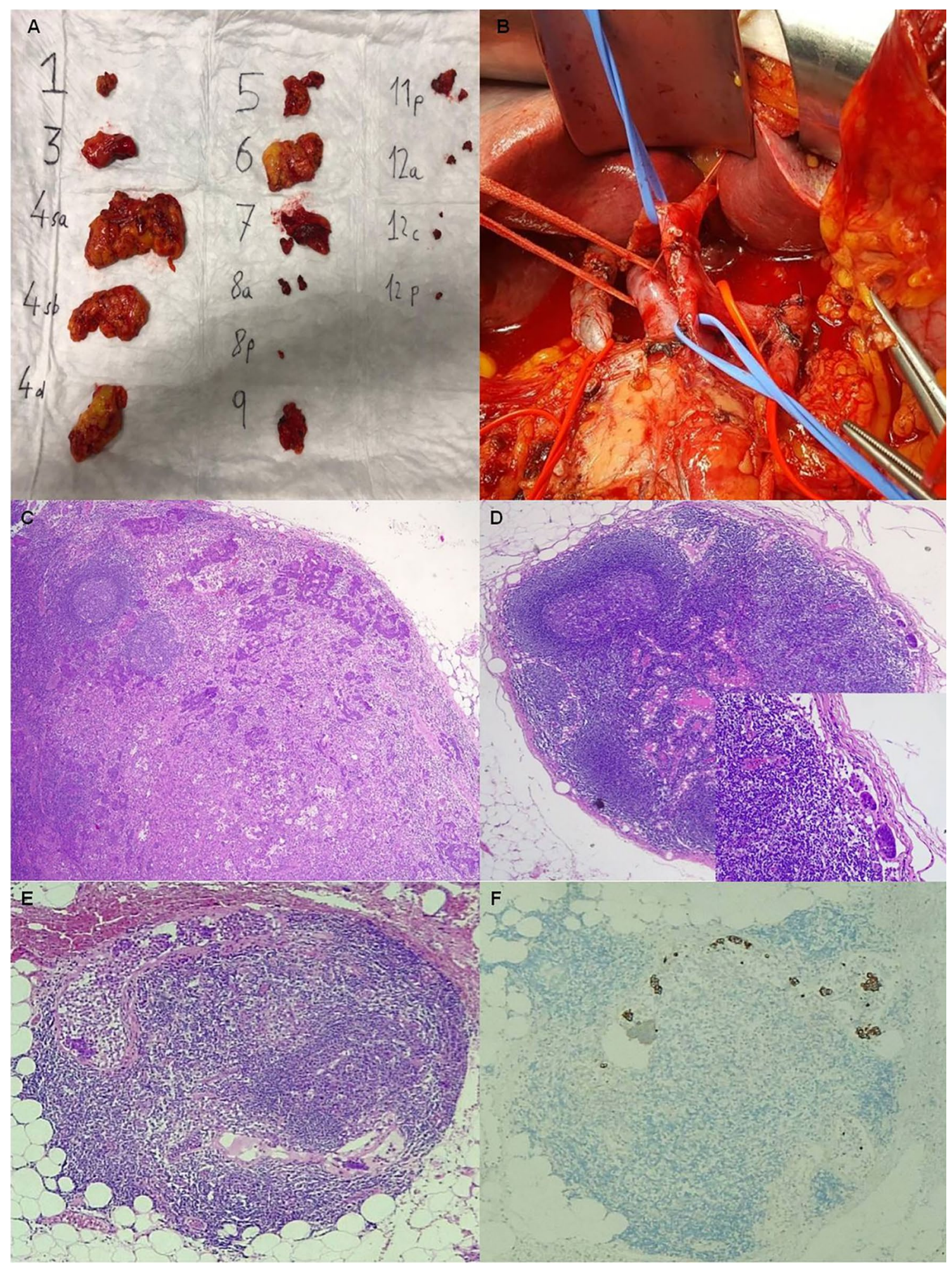

Fig. 1 Lymph node harvesting according to the Japanese Gastric Cancer Protocol. a The different lymph node stations are separated and picked up. b The surgical bed after lymph nodes removal. c One of the two metastatic lymph nodes occurring far from the site of the primary tumor (i.e. in number 3 station). d-d, inset A very small metastasis $(140 \mu \mathrm{m})$ found in a $1.3 \mathrm{~mm}$ sized lymph node of the number 6 station. e-f The metastasis $(0.45 \mathrm{~mm})$ identified in the smallest lymph node $(0.7 \mathrm{~mm})$ harvested. $\mathbf{c}-\mathbf{e}$ haematoxylin and eosin stain; $\mathbf{f}$ $8 / 18$ cytokeratin stain. Original magnification: $\mathbf{c}-\mathbf{f} \times 5$; $\mathbf{d}$ inset: $\times 40$ 
As a second step, in the retrospective phase, we enrolled a control group represented by the last ten patients who have undergone a gastrectomy plus D1 or D2 lymphadenectomy before the beginning of the SARS-COV2 pandemic. The control group encompassed 8 males and 2 females with a median age of 77.9 years old (range 60-92 years). The operative procedure in the control group had been performed by the same surgeon but LNs had been harvested by different pathologists following the classic manual method in the grossing room (not in the OR) on FF specimens (not on fresh tissue); the JGCA protocol was applied only in five patients.

We compared the number and maximum diameter of LNs collected, as well as the time required for mapping, in the two groups. The package Systat 4 software was used for the statistical analysis with a $p$ value $<0.05$ being considered statistically significant.

According to the results of the statistical analysis, the mean number of LNs identified by the surgeon/pathologist team by on site evaluation method in OR following the JGCA protocol was greater than the standard procedure carried out by the pathologist alone ( 86 versus 20,6 ), with $p<0.05$. In the study group also the smallest LNs were identified and harvested $(0.7$ versus $5 \mathrm{~mm}, p<0.05)$. In one patient we recognized 3 metastatic LNs out of $145 \mathrm{LN}$ examined, 2 in site far from the tumor and 1 with a very small $(140 \mu \mathrm{m})$ metastasis (Fig. 1c-f). Unfortunately, there were no statistically significant differences in the number of LNs between the two groups based on a comparison of the lymphatic compartments $(p=0.6)$; this was probably due to the small number of samples following the JGCA protocol in the control group. As far as the timing for LNs harvesting and mapping was concerned, the procedure was shorter in the control group (30 versus $45 \mathrm{~min}$ ).

Studies have demonstrated the importance of both the numerical and anatomic extent of LNs assessment in its impact on the proper identification of LN metastases. [9] The site of metastasis marks the regional spread of the disease and portents increased risk of recurrence and poorer prognosis [8]. The surgical and pathological skills and techniques heavily impact on the number of retrieved LNs, justifying an accurate training of dedicated medical personnel with a learning curve in particular in low-volume centers. Surgeons with thorough anatomical knowledge of the various LN stations and surgical experience should perform the gastrectomy, and skilled pathologists should examine and count LNs providing a complete pathological evaluation $[10,11]$. In fact, the lymphatic system involved in GC is complex since it is three-dimensional and multi-directional. Starting from the belief that the team approach is absolutely essential in improving the quality of patient care, we decided to put together surgeon and pathologist. By cooperation, their own vigilance and insight were stimulated acting as an extra-motive for a more careful analysis of the specimen to "search for the unlikely". In fact, there are things that only the surgeon experienced but similarly there are notions that only the pathologist knows. This communication aims to illustrate and prove the feasibility and utility of a surgeon/pathologist team approach for facilitating and improving LNs sampling. The disadvantages include the need of a pathology in the OR and the time for sampling. However, if considering the higher number of LNs harvested, including the smallest ones, we should not regard these tools as a drawback because the workload is only related to the sampling. From the foregoing, it is obvious that the merits of improved surgeon-pathologist collaboration far outweigh whatever disadvantages that may arise from such interactions. Nevertheless, since a larger number of patients are necessary to confirm these preliminary results and to assess the impact of our technique on oncological outcome of the patients, this communication can serve as proof-of-concept for a larger study, that is still ongoing in our Institutions.

Author contributions All authors contributed to the study conception and design. Patients and data collection was performed by Dr. Marco Arganini and Dr. Bruno Perotti. Data analysis was performed by Dr.ssa Maria Raffaella Ambrosio. The first draft of the manuscript was written by Dr. Marco Arganini, Dr.ssa Maria Raffaella Ambrosio and Dr. Andrea Cavazzana. All authors commented on previous versions of the manuscript. All authors read and approved the final manuscript.

\section{Declarations}

Conflict of interest The authors have no conflicts of interest to declare that are relevant to the content of this article. All authors certify that they have no affiliations with or involvement in any organization or entity with any financial interest or non-financial interest in the subject matter or materials discussed in this manuscript. The authors have no financial or proprietary interests in any material discussed in this article. Authors are responsible for correctness of the statements provided in this manuscript.

Research involving human participants and/or animals The research did not involve human partecipants or animals.

Informed consent Informed written consent was obtained by the patients.

\section{References}

1. Eshuis WJ, van Barge Henegouwen MI, Draaisma WA, Gisbert SS (2018) Compliance to D2 lymphadenectomy in laparoscopic gastrectomy. Updates Surg 70:197-205

2. Zhang N, Bai H, Deng J et al (2020) Impact of examined lymph node count on staging and long-term survival of patients with node-negative stage III gastric cancer: a retrospective study using a Chinese multi-institutional registry with Surveillance, Epidemiology, and End Results (SEER) data validation. Ann Transl Med 8:1075-1091 
3. Kano K, Yamada T, Yamamoto K et al (2020) Association between lymph node ratio and survival in patients with pathological stage II/III gastric cancer. Ann Surg Oncol 27:4235-4247

4. Zhang YX, Yang K (2020) Significance of nodal dissection and nodal positivity in gastric cancer. Transl Gastroenterol Hepatol 5(5): 17

5. Pan S, Wang P, Xing Y et al (2019) Retrieved lymph nodes from different anatomic groups in gastric cancer: a proposed optimal number, comparison with other nodal classification strategies and its impact on prognosis. Cancer Commun 39:49

6. Kinami S, Ohnishi T, Nakamura N et al (2020) Efficacy of the fatdissociation method for nodal harvesting in gastric cancer. World J Gastrointest Surg 12:277-286

7. Baiocchi GL, Molfino S, Molteni B et al (2020) Fluorescenceguided lymphadenectomy in gastric cancer: a prospective western series. Updates Surg 72:761-772

8. Japanese Gastric Cancer Association (2018) Japanese gastric cancer treatment guidelines 2018 (5th edition). Gastric Cancer. https://doi.org/10.1007/s10120-020-01042-y
9. Macalindong SS, Kim KH, Nam BH (2018) Effect of total number of harvested lymph nodes on survival outcomes after curative resection for gastric adenocarcinoma: findings from an eastern high-volume gastric cancer center. BMC Cancer 18:73. https:// doi.org/10.1186/s12885-017-3872-6

10. Bencivenga M, Verlato G, Mengardo V, Weindelmayer J, Allum WH (2018) Do all the European surgeons perform the same D2? The need of D2 audit in Europe. Updates Surg 70:189-195

11. da Costa PM, Lages P, Onofre S, Ribeiro RM (2020) The impact of negative lymph nodes in the survival outcomes of $\mathrm{pN}+$ patients following radical gastrectomy: the inverse lymph node ratio as a better score to study negative lymph nodes. Updates Surg 72:1031-1040

Publisher's Note Springer Nature remains neutral with regard to jurisdictional claims in published maps and institutional affiliations. 